\title{
STRUCTURE OF NONLINEAR WHISTLERS MOVING THROUGH PLASMA AT AN ANGLE TO THE MAGNETIC FIELD
}

\author{
G.N. Kichigin \\ Institute of Solar-Terrestrial Physics SB RAS, \\ Irkutsk, Russia,king@iszf.irk.ru
}

\begin{abstract}
The paper presents solutions of two-fluid magnetic hydrodynamics equations describing smallscale fast magnetosonic stable waves - nonlinear whistlers moving in a cold magnetized plasma at an angle $\alpha$ to the external magnetic field. At the fixed angle $\alpha$, the Alfvén Mach number of the whistlers has a narrow range of allowed values. It has been found that when passing from extremely small Mach numbers to extremely large ones, amplitudes and spatial structure of wave velocity components and whistler magnetic field
\end{abstract}

change significantly. The range of angles of the motion direction of whistlers with respect to direction of the external magnetic field vector is determined. Within this range, the obtained approximate analytical and numerical solutions are in satisfactory agreement.

Keywords: magnetosonic waves; nonlinear whistlers.

\section{INTRODUCTION}

This paper studies stable nonlinear waves - whistlers propagating at an angle $\alpha(\alpha \neq \pi / 2)$ to a constant uniform magnetic field in a cold collisionless plasma. The emphasis is on fast magnetic sound (FMS) waves, which under the condition $\omega_{\mathrm{He}}<<\omega_{\mathrm{pe}}$ have a frequency $\omega_{\mathrm{Hi}}<<\omega<\omega_{\mathrm{He}} \cos \alpha$ and wavelengths of order of electron inertial length $c / \omega_{\mathrm{pe}}$ (here $\omega_{\mathrm{Hi}}$ is the ion cyclotron frequency, $\omega_{\mathrm{He}}$ is the electron cyclotron frequency, $\omega_{\mathrm{pe}}$ is the electron plasma frequency, $c$ is the speed of light). FMS waves in this frequency range have several names: whistling atmospherics, whistler waves, helicons, and whistlers [Akhiezer et al., 1974; Gershman, Ugarov, 1960]. In this paper, these waves are called whistlers. Whistlers are quite often observed in many phenomena that occur in the magnetized cosmic plasma. For example, whistlers exciting in near-Earth plasma due to continuous lightning discharges in Earth's atmosphere [Gershman, Ugarov, 1960] are constantly recorded by ground-based radio receivers. Another example is the recent discovery that whistlers play an essential role in the formation of the structure of collisionless shocks [Balogh, Treumann, 2013], in particular, near-Earth shocks [Wilson III, 2016].

As follows from the results obtained by Saffman [1961], for stable nonlinear whistlers moving strictly along lines of the external magnetic field, the wave magnetic field has two components comparable in magnitude and transverse to the direction of wave propagation, and the wave magnetic field vector rotates in the same transverse plane. The characteristic spatial scale of whistlers is $\sim c / \omega_{\mathrm{pe}}$. The velocity of the whistler traveling strictly along the external magnetic field is by an order of magnitude higher than the Alfvén velocity in an unperturbed plasma, and the amplitude of the transverse components of the whistler wave field at its maximum velocities is by an order of magnitude higher than the strength of the external magnetic field. This study shows that whistlers moving at an angle to the magnetic field have a similar structure.

\section{FORMULATION OF THE PROBLEM AND BASIC EQUATIONS}

Consider the problem of the structure of a nonlinear disturbance in a cold magnetized plasma, which moves with a velocity $U_{0}$ at an angle $\alpha$ to the $H_{B}$ external stationary magnetic field. Use the frame of reference associated with the disturbance wave. Suppose that the motion of plasma particles is nonrelativistic. Restrict ourselves to a one-dimensional case, i.e. assume that all variables depend only on the coordinate $x$. Assume in addition that in an unperturbed plasma the external homogeneous magnetic field has components $H_{x 0}=H_{B} \cos \alpha$ and $H_{z 0}=H_{B} \sin \alpha$. In the approximation of two-fluid magnetohydrodynamics, assuming that, when the condition $\omega_{\mathrm{He}}<\omega_{\mathrm{pe}}$ holds, the plasma is quasineutral, and the effects associated with the finite pressure of the background plasma can be ignored, obtain the following relations for stable waves in a hydrogen plasma [Karpman, 1973; Tidman, Krall, 1971; Sagdeyev, 1964; Kellogg, 1964; Aldam, Allen, 1958]:

$$
\begin{aligned}
& m_{\mathrm{p}} U d \mathbf{v}_{\mathrm{p}} / d x=e \mathbf{E}+e\left[\mathbf{v}_{\mathrm{p}} \times \mathbf{H}\right] / c, \\
& m_{\mathrm{e}} U d \mathbf{v}_{\mathrm{e}} / d x=-e \mathbf{E}-e\left[\mathbf{v}_{\mathrm{e}} \times \mathbf{H}\right] / c, \\
& \nabla \times \mathbf{H}=4 \pi e n\left(\mathbf{v}_{\mathrm{p}}-\mathbf{v}_{\mathrm{e}}\right) / c, \nabla \times \mathbf{E}=0, \\
& n_{\mathrm{e}}(x)=n_{\mathrm{p}}(x)=n(x), U_{\mathrm{p}}(x)=U_{\mathrm{e}}(x)=U(x), \\
& n(x) U(x)=\text { const }=j, \\
& H_{x}=\text { const }=H_{x 0} \equiv H_{0}, E_{y}=\text { const, } \\
& E_{z}=\text { const, } E_{x}=-[1 /(m c)]\left[\left(m_{\mathrm{e}} \mathbf{v}_{\mathrm{p}}+m_{\mathrm{p}} \mathbf{v}_{\mathrm{e}}\right) \times \mathbf{H}\right]_{x} .
\end{aligned}
$$

Here $\mathbf{E}$ and $\mathbf{H}$ are the electric and magnetic field vectors, $U$ is the $x$ plasma particle velocity component, $n$ is the particle density, $\mathbf{v}_{\mathrm{p}}$ and $m_{\mathrm{p}}$ are the proton velocity vector and mass, $\mathbf{v}_{\mathrm{e}}$ and $m_{\mathrm{e}}$ is the electron velocity vector and mass, $e$ is the electron charge, $m=m_{\mathrm{p}}+m_{\mathrm{e}}$. The above relations yield the following momentum conservation laws: 


$$
\begin{aligned}
& m U+\left(H_{y}^{2}+H_{z}^{2}\right) /(8 \pi j)=p, \\
& m_{\mathrm{p}} V_{\mathrm{p}}+m_{\mathrm{e}} V_{\mathrm{e}}-H_{0} H_{y} /(4 \pi j)=p_{1}, \\
& m_{\mathrm{p}} W_{\mathrm{p}}+m_{\mathrm{e}} W_{\mathrm{e}}-H_{0} H_{z} /(4 \pi j)=p_{2},
\end{aligned}
$$

where $p, p_{1}$, and $p_{2}$ are constants, and $V$ and $W$ denote $y$ and $z$ particle velocity components respectively. Specifying the variables in the unperturbed plasma $(x \rightarrow-\infty)$

$$
\begin{aligned}
& H_{y}=0, H_{z}=H_{z 0}, d \mathbf{H} / d x=0, E_{x}=E_{z}=0, \\
& E_{y}=U_{0} H_{z 0} / c, U=U_{0}, n=n_{0}, V_{\mathrm{p}}=V_{\mathrm{e}}=W_{\mathrm{p}}=W_{\mathrm{e}}=0,
\end{aligned}
$$

get for constants

$$
p=m U_{0}+H_{z 0}{ }^{2} /(8 \pi j), p_{1}=0, p_{2}=-H_{0} H_{z 0} /(4 \pi j) .
$$

Introduce dimensionless variables and parameters. Normalize the coordinate by the length $c / \omega_{\mathrm{p}}$ and symbolize as

$$
\xi=x \omega_{\mathrm{p}} / c,
$$

where $\omega_{\mathrm{p}}=\sqrt{4 \pi n_{0} e^{2} m /\left(m_{\mathrm{e}} m_{\mathrm{p}}\right)} \approx \omega_{\mathrm{pe}}$, designate the dimensionless time as

$$
\tau=\omega_{\mathrm{g}} t
$$

where $\omega_{\mathrm{g}}=e H_{B} /\left(c \sqrt{m_{\mathrm{e}} m_{\mathrm{p}}}\right)$. Denote the dimensionless quantities of the magnetic field components, normalized to $H_{B}$, by $h$ (with corresponding index); the $x, y$, and $z$ particle velocity components, normalized to $V_{\mathrm{A}}$, by $u, v$, and $w$ respectively (here $V_{\mathrm{A}}=H_{B} / \sqrt{4 \pi n_{0} m}$ is the Alfvén wave velocity in the unperturbed plasma). Designate the dimensionless potential as $\psi=2 \mathrm{e} \varphi /\left(m U_{0}{ }^{2}\right)$. Use the following notations for dimensionless parameters: Alfvén Mach number $M=U_{0} / V_{\mathrm{A}}, h_{0}=H_{0} / H_{B}=\cos \alpha$, root of the particle mass ratio $\mu=\sqrt{m_{\mathrm{p}} / m_{\mathrm{e}}}, R=\mu+1 / \mu$.

Given that $u=d \xi / d \tau$, the above relations yield a system of equations for finding dimensionless components of particle velocities and magnetic field components in a disturbance wave:

$$
\begin{aligned}
& d v_{\mathrm{e}} / d \tau=\mu\left(u h_{z}-M h_{z 0}-w_{\mathrm{e}} h_{0}\right), \\
& d w_{\mathrm{e}} / d \tau=\mu\left(v_{\mathrm{e}} h_{0}-u h_{y}\right), \\
& d h_{y} / d \tau=\left[h_{0}\left(h_{z}-h_{z 0}\right)-M w_{\mathrm{e}}\right] / \mu, \\
& d h_{\mathrm{z}} / d \tau=\left(M v_{\mathrm{e}}-h_{0} h_{y}\right) / \mu, \\
& u=M+\left(h_{z 0}{ }^{2}-h_{y}{ }^{2}-h_{\mathrm{z}}{ }^{2}\right) /(2 M), \\
& v_{\mathrm{p}}=-v_{\mathrm{e}} / \mu+\left(1+\mu^{-2}\right) h_{0} h_{y} / M, \\
& w_{\mathrm{p}}=-w_{\mathrm{e}} / \mu+\left(1+\mu^{-2}\right) h_{0}\left(h_{z}-h_{z 0}\right) / M .
\end{aligned}
$$

System of equations (1)-(4) in view of (5) is sufficient to find magnetic field and electron velocity components. Equations (6)-(7) allow us to find proton velocity components.

System of equations (1)-(7) can be solved with numerical methods for solving systems of differential equations. To solve it, the Runge-Kutta method was employed. The accuracy of the numerical solutions was checked by comparing them with analytical formulas for two particular cases: 1) a wave moving strictly along $(\alpha=0)$ the external magnetic field [Saffman, 1961]; 2) a wave moving strictly across $(\alpha=\pi / 2)$ the external magnetic field [Aldam, Allen, 1958]. The comparison has shown that the calculation accuracy was at least $10^{-5}$.

\section{APPROXIMATE \\ ANALYTICAL SOLUTIONS OF SYSTEM OF EQUATIONS (1)-(7)}

Taking into account the results obtained by Saffman [1961], it can be assumed that for oblique FMS waves traveling at the maximum possible velocity almost along the external field $\left(h_{z 0}<<1, h_{0} \approx 1\right)$, the amplitude of the transverse components of the wave magnetic field considerably (tens of times) exceeds the strength of the $H_{B}$ external magnetic field. Using this fact, in the first approximation we can ignore the contribution of the transverse component of the external magnetic field $h_{z 0}$ to the total field and can put $h_{z 0}=0$ as the boundary condition (if $x \rightarrow-\infty$ ). With this simplification, system of equations (1)-(7) has an analytic solution. Indeed, if $h_{z 0}=0$, (1)-(7) yield the following relations:

$$
\begin{aligned}
& w_{\mathrm{e}}{ }^{2}+v_{\mathrm{e}}{ }^{2}=\mu^{2}\left(M^{2}-u^{2}\right), w_{\mathrm{p}}{ }^{2}+v_{\mathrm{p}}{ }^{2}=\left(M^{2}-u^{2}\right) / \mu^{2}, \\
& w_{\mathrm{e}} h_{y}-v_{\mathrm{e}} h_{z}=-\mu^{2}\left(w_{\mathrm{p}} h_{y}-v_{\mathrm{p}} h_{z}\right)= \\
& =\mu M\left(w_{\mathrm{e}} v_{\mathrm{p}}-w_{\mathrm{p}} v_{\mathrm{e}}\right) /\left(R h_{0}\right)=\mu d u / d \tau, \\
& \left(w_{\mathrm{e}} v_{\mathrm{p}}-w_{\mathrm{p}} v_{y}\right)^{2}+\left(v_{\mathrm{e}} v_{\mathrm{p}}+w_{\mathrm{e}} w_{\mathrm{p}}\right)^{2}= \\
& =\left(w_{\mathrm{e}}{ }^{2}+v_{\mathrm{e}}{ }^{2}\right)\left(w_{\mathrm{p}}{ }^{2}+v_{\mathrm{p}}{ }^{2}\right)=\left(M^{2}-u^{2}\right)^{2},
\end{aligned}
$$

which can be reduced to one differential equation for $u$ :

$$
d u / d \tau=(M-u)\left[4 M^{2}-h_{0}{ }^{2} R^{2}-2 M(M-u)\right]^{1 / 2} .
$$

Hence, taking into account the boundary conditions, after integration we get

$$
\begin{aligned}
& u=M-2 \lambda^{2} \operatorname{sech}^{2}(\lambda \tau) / M, \\
& b=\sqrt{h_{y}^{2}+h_{z}^{2}}=2 \lambda \operatorname{sech}(\lambda \tau),
\end{aligned}
$$

where $\lambda=\sqrt{M^{2}-h_{0}^{2}\left(\mu+\mu^{-1}\right)^{2} / 4}$. The difference from the solution derived by Saffman [1961] is that the parameter $\lambda$ depends on $h_{0}=\cos \alpha$. Given $h_{0}=\cos \alpha=1$, formulas (8)-(9) coincide with the formulas obtained by Saffman [1961]. As in [Saffman, 1961], in this solution for each given angle $\alpha$, the wave Mach number has a limited range of possible values:

$$
(\mu+1 / \mu) \cos \alpha / 2<M<(\mu+1 / \mu) \cos \alpha / \sqrt{2} .
$$
as

Represent the field components through its modulus $b$

$$
h_{z}=b \cos \beta, h_{y}=b \sin \beta .
$$

Find the argument $\beta$, taking into account formulas (1)-(4): $\beta=\beta(\tau)=\left(\mu^{2}-1\right) h_{0} /(2 \mu) \tau$. Thus, the final formulas for the magnetic field components are

$$
\begin{aligned}
& h_{z}=2 \lambda \cos \beta \operatorname{sech}(\lambda \tau), \\
& h_{y}=2 \lambda \sin \beta \operatorname{sech}(\lambda \tau),
\end{aligned}
$$

where $\beta=\beta(\tau)=\left(\mu^{2}-1\right) h_{0} /(2 \mu) \tau=\gamma \tau\left(\gamma=h_{0}(\mu-1 / \mu) / 2\right)$. With these formulas, equations (3)-(4) give expressions for the transverse components of electron velocities; and (6)-(7), for the transverse components of ion velocities. It remains to find the whistler potential profile; to do this, use the formula

$d \varphi / d x=[1 /(m c)]\left[\left(m_{\mathrm{e}} \mathbf{v}_{\mathrm{p}}+m_{\mathrm{p}} \mathbf{v}_{\mathrm{e}}\right) \times \mathbf{H}\right]_{x}$. Being dimensionless, this formula takes the form

$$
\begin{aligned}
& c(d \varphi / d x) /\left(H V_{\mathrm{A}}\right)=m M^{2}(d \psi / d \xi) /\left[2\left(m_{\mathrm{e}} m_{\mathrm{p}}\right)^{1 / 2}\right]= \\
& =\left(m_{\mathrm{e}} / m\right)\left(v_{\mathrm{p}} h_{z}-w_{\mathrm{p}} h_{y}\right)+\left(m_{\mathrm{p}} / m\right)\left(v_{\mathrm{e}} h_{z}-w_{\mathrm{e}} h_{y}\right) .
\end{aligned}
$$

Using here the relations 


$$
\begin{aligned}
& v_{\mathrm{p}} h_{\mathrm{z}}-w_{\mathrm{p}} h_{\mathrm{y}}=-\left(m_{\mathrm{e}} / m_{\mathrm{p}}\right)\left(v_{\mathrm{e}} h_{z}-w_{\mathrm{e}} h_{y}\right)= \\
& =-\left(m_{\mathrm{e}} / m_{\mathrm{p}}\right)^{1 / 2} u(d u / d \xi),
\end{aligned}
$$

get

$$
d \psi / d \xi=-\left[\left(\mu^{2}-1\right) /\left(\mu^{2}+1\right)\right]\left[d\left(u^{2} / M^{2}\right) / d \xi\right] .
$$

Taking into account the boundary conditions and assuming that $\mu^{2} \gg>1$, have $\psi=1-u^{2} / M^{2}$. It is easy to see that the whistler potential is maximum $\psi=\psi_{m}$, and the $x$ particle velocity component is minimum $u=0$ at $\tau=0$ :

$$
\begin{aligned}
& \psi_{\mathrm{m}}=\psi(0)=1-\left(1-2 \lambda^{2} / M^{2}\right)^{2}=4\left(1-\lambda^{2} / M^{2}\right) \lambda^{2} / M^{2} \\
& =\left(h_{0}{ }^{2} R^{2} / M^{2}\right)\left[1-h_{0}{ }^{2} R^{2} /\left(4 M^{2}\right)\right] .
\end{aligned}
$$

The maximum potential is zero at the minimum Mach number $M=h_{0} R / 2$ and has a limit value $\psi=1$ at the maximum Mach number $M=h_{0} R / \sqrt{2}$. An interesting feature of the results should be noted: the minimum propagation velocity of the nonlinear whistlers considered, with amplitudes of the magnetic field components and potential being infinitely small, is equal to the maximum phase velocity FMS waves with wavelengths $\sim c / \omega_{\mathrm{pe}}$ have in the linear approximation.

To complete the calculations, from (8) derive a formula that relates $\xi$ to $\tau$ :

$$
\xi(\tau)=M \tau-2 \lambda \operatorname{th}(\lambda \tau) / M \text {. }
$$

With this formula, the relations for the potential, magnetic field and plasma particle velocity components completely determine the structure of the whistlers. Figures 1-3 present spatial profiles of magnetic field components and the potential for two Mach numbers, one of which, $M=27.56$, is nearly maximum; the other, $M=22.44$, nearly minimum. These Figures show that as the Mach number decreases, the spatial structure of potential and magnetic field component profiles changes, and the amplitudes of magnetic field components and potential decrease. The spatial profile of the potential (Figure 3) takes the shape of a solitary wave, or soliton. The potential profile for ion-acoustic soliton [Akhiezer et al., 1974; Sagdeyev, 1964] and the potential and magnetic field profiles for a strictly transverse magnetosonic soliton [Akhiezer et al., 1974; Karpman, 1973; Tidman, Krall, 1971; Sagdeyev, 1964; Aldam, Allen, 1958] have a similar shape.

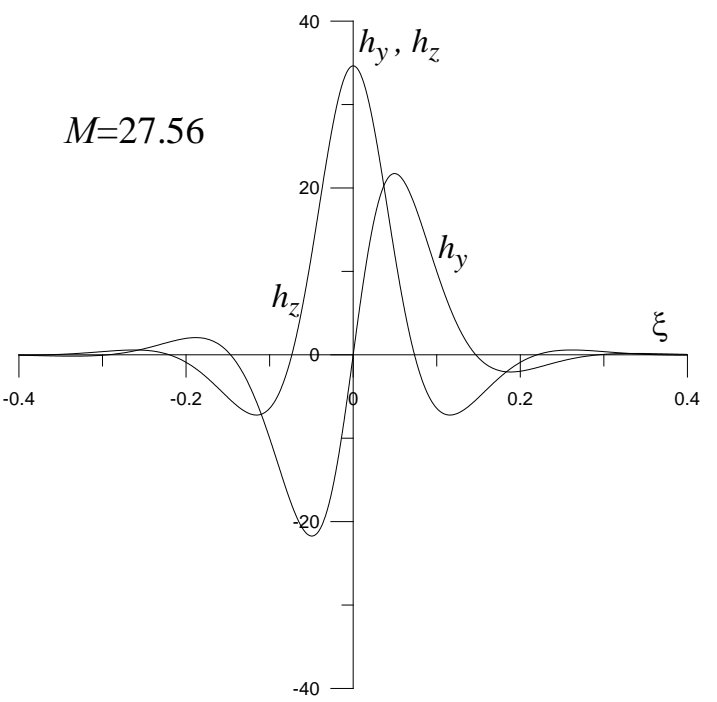

Figure 1. Profiles of whistler magnetic field components $h_{y}, h_{z}$ for $M=27.56$

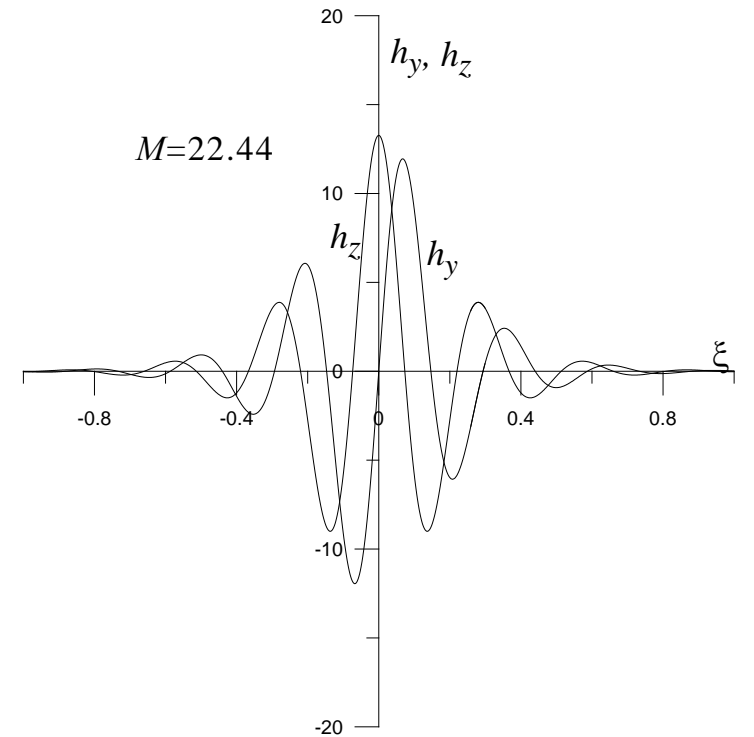

Figure 2. Profiles of whistler magnetic field components $h_{y}, h_{z}$ for $M=22.44$

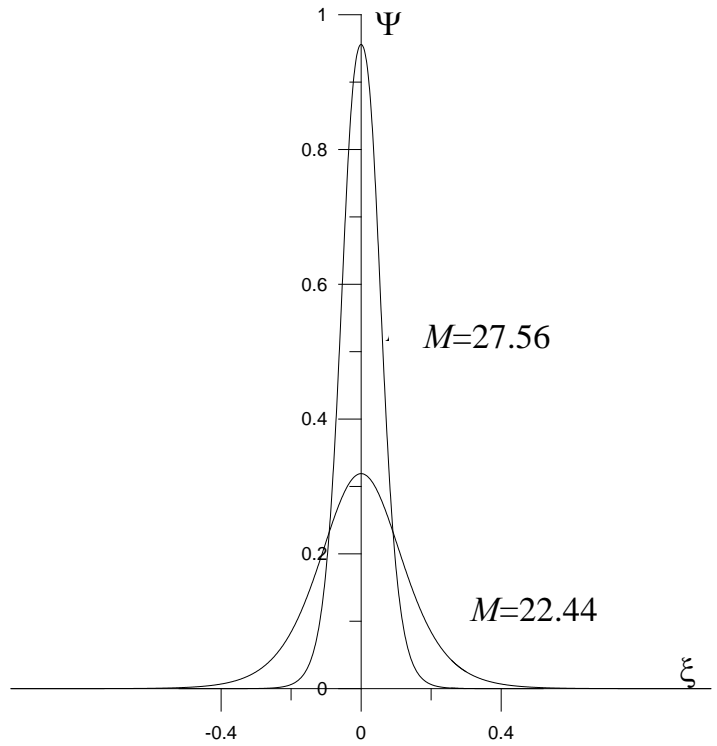

Figure 3. Spatial profiles of the whistler potential for $M=27.56$ and $M=22.44$

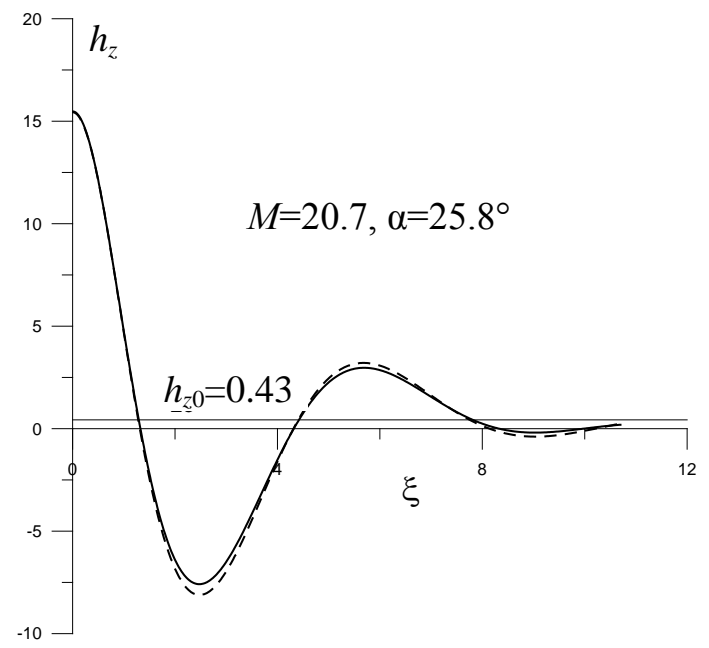

Figure 4. Spatial profiles of the $h_{z}$ component obtained from numerical calculations (solid line) and calculated from formula (9) (dashed line) for $M=20.7, h_{0}=0.9$, and $h_{z 0}=0.43$ 
To determine the limits of applicability, our approximate solutions were compared with solutions derived from numerical calculations. Results of this comparison are presented in Figure 4, which shows only half of the $h_{z}$ profile symmetric about the point $\xi=0$. The closest agreement is observed for Mach numbers close to extremely large values for which the comparison allowed us to establish the range of applicability of our approximation - an angle range of $0^{\circ}<\alpha<80^{\circ}\left(1>h_{0}>0.2\right)$. In this range, analytical solutions can be used for describing the magnetic field structure in oblique whistlers.

I believe that the nonlinear solutions obtained for whistlers determine the nonlinear structure of the shock discontinuity of supercritical oblique collisionless shock waves (CSW), at the front of which there are magnetic field jumps of sufficiently high amplitude [Balogh, Treumann, 2013]. This refers to CSWs propagating in space plasma, in particular, interplanetary and nearEarth CSWs. The spatial structure of the magnetic field jump at the CSW front is assumed to have the form of a nonlinear whistler. This assumption is confirmed by the numerical experiment described by Krasnoselskikh et al. [2002]. The authors emphasize the essential role played by nonlinear whistlers in the formation of the front of strong CSWs.

\section{MAIN RESULTS AND CONCLUSIONS}

1. Solutions have been found for two-fluid hydrodynamics equations describing small-scale fast magnetosonic nonlinear waves - whistlers moving through plasma at an angle $\alpha$ to the external magnetic field.

2. At a fixed angle $\alpha$, the Alfvén Mach number $M$ of whistlers has a small range of allowed values: $\mu \cos \alpha / 2<M<\mu \cos \alpha / \sqrt{2}$. When passing from extremely small Mach numbers to extremely large ones, the amplitude and spatial structure of the potential of wave velocity components and whistler magnetic field change significantly.

3. The range of angles of the motion direction of whistlers relative to the direction of the external magnetic field vector has been determined. Within this range, the obtained approximate analytical and numerical solutions are in satisfactory agreement. For whistler velocities close to the highest values $M \approx \mu \cos \alpha / \sqrt{2}$ this is the range of angles $0^{\circ}<\alpha<80^{\circ}$.

The work was carried out as part of Project II.16.1.3 of SB RAS Program for Fundamental Scientific Research II.16.1 with budgetary funding of Basic Research program II.16.

\section{REFERENCES}

Akhiezer A.I., Akhiezer I.A., Polovin R.V., Sitenko A.G., Stepanov K.N. Elektrodinamika plazmy [Plasma Electrodynamics]. Moscow, Nauka Publ., 1974, 719 p. (In Russian).

Aldam J., Allen J. The structure of strong collision-free hydromagnetic waves. The Philosophical Magazine: A Journal of Theoretical Experimental and Applied Physics. Ser. 8. 1958, vol. 3, pp. 448-455.
Balogh A., Treumann R.A. Physics of Collisionless Shocks. New York, Springer Science Business Media, 2013, DOI: 10.1007/978-1-4614-6099-2.

Gershman B.I., Ugarov V.A. Propagation and generation of low-frequency electromagnetic waves in the upper atmosphere. Uspekhi fizicheskikh nauk [Physics-Uspekhi (Adv. Phys. Sci.)]. 1960, vol. LXXII, iss. 2, pp. 235-271.

Karpman V.I. Nelineinye volny $v$ dispergiruyushchikh sredakh [Non-Linear Waves in Dispersive Media]. Moscow, Nauka Publ., 1973, 175 p. (In Russian).

Sagdeyev R.Z. Collective processes and shock waves in lowdensity plasma. Voprosy teorii plazmy [Plasma Theory Matters]. Moscow, Atomizdat Publ., 1964, vol. 4, p. 20. (In Russian).

Kellogg P.J. Solitary waves in a cold collisionless plasma. Physics of Fluids. 1964. V. 7, iss.10, pp. 1555-1571.

Krasnoselskikh V.V., Lembège B., Savoini P., Lobzin V.V. Nonstationarity of strong collisionless quasiperpendicular shocks: Theory and full particle numerical simulations. Phys. Plasmas. 2002, vol. 9, pp. 1192-1209. DOI: 10.1063/1.1457465.

Saffman P.G. Propagation of a solitary wave along a magnetic field in a cold collision-free plasma. J. Fluids Mech. 1961, vol. 11, pp. 16-20.

Tidman D.F., Krall N.A. Shock Waves in Collisionless Plasmas. New York-London-Sidney-Toronto, WileyInterscience; A Division of John Wiley \& Sons Inc., 1971, 166 p.

Wilson III L.B. Low Frequency Waves at and Upstream of Collisionless Shocks. Low Frequency Waves in Space Plasmas. WiLey, 2016, pp. 269-292.

How to cite this article

Kichigin G.N. Structure of nonlinear whistlers moving through plasma at an angle to the magnetic field. Solar-Terrestrial Physics. 2018, vol. 4, no. 1, pp. 25-28. DOI: 10.12737/stp-41201803. 